\title{
Using afterimages for orientation and color to explore mechanisms of visual filling-in
}

\author{
GREGORY FRANCIS and WADE SCHOONVELD \\ Purdue University, West Lafayette, Indiana
}

\begin{abstract}
Simulations of Grossberg's FACADE model of visual perception have previously been used to explain afterimage percepts produced by viewing a sequence of orthogonally oriented gratings. Additional simulations of the model are now used to predict new afterimage percepts. One simulation emphasizes that the afterimage percepts are the result of orientation afterresponses and color afterresponses that interact at a filling-in stage. We report experimental data that agree with FACADE's prediction. A second simulation emphasizes the properties of the model's filling-in stage and predicts a situation where the afterimage percept should not appear. We report experimental data indicating that this model prediction is incorrect. We argue that the model is unable to account for this result unless the filling-in stage mechanisms are different from a diffusive-type process. We propose an alternative mechanism, and simulations demonstrate the system's ability to account for the afterimage data.
\end{abstract}

Part of the construction of visual percepts seems to involve a filling-in process that computes information about perceived colors and brightness across surfaces (Gerrits \& Vendrik, 1970; Pessoa, Thompson, \& Noë, 1998). Illusions such as neon color spreading and the water-color illusion (da Pos \& Bressan, 2003; Pinna, Brelstaff, \& Spillmann, 2001) have been taken as direct evidence of filling-in. Filling-in processes have also been invoked to explain a variety of other percepts, including brightness perception (Grossberg \& Todorović, 1988; Todorović, 1987), properties of McCollough afterimages (Broerse, Vladusich, \& O’Shea, 1999; Grossberg, Hwang, \& Mingolla, 2002), properties of color complement afterimages (Shimojo, Kamitani, \& Nishida, 2001), and some aspects of 3-D perception (Grossberg, 1997).

Although there is agreement in some circles that fillingin mechanisms exist, the details of those mechanisms are unclear. The standard view is that the filling-in process is similar to an isotropic diffusion of information, from edges to interiors of regions (Gerrits \& Vendrik, 1970). Consistent with this idea, Paradiso and Nakayama (1991) demonstrated that a circular mask appeared to block the diffusive spread of brightness information (see also Arrington, 1994; Stoper \& Mansfield, 1978). Neurophysiological evidence on filling-in mechanisms has been unclear. Early reports on the representation of edge and surface information in area V1 of monkeys suggested that whereas edges were coded by orientation-sensitive neurons, color-sensitive neurons were not orientationally

G. F. was supported by a fellowship at the Hanse Wissenschaftskolleg, Delmenhorst, Germany. Correspondence concerning this article should be addressed to G. Francis, Department of Psychological Sciences, 703 Third Street, West Lafayette, IN 47907-2004 (e-mail: gfrancis@ psych.purdue.edu). tuned (e.g., Livingstone \& Hubel, 1984). This has been taken as evidence for an anatomical segregation of form (edges) and surface (color) information in visual cortex. Komatsu, Kinoshita, and Murakami (2000) measured activity from cells responding to a homogeneous pattern that covered the blind spot, which implies the presence of a filling-in mechanism. Generally, these findings are consistent with a diffusive filling-in mechanism for surface brightness and color. However, Friedman, Zhou, and von der Heydt (2003) report evidence that many colorsensitive cells are also highly orientation selective. Friedman et al. argue that current neurophysiological evidence no longer supports the hypothesized anatomical separation of form and color information. The orientation sensitivity of neurons supporting a filling-in mechanism seems contrary to isotropic diffusion of color and brightness that is part of most filling-in theories.

Perhaps the most advanced model that incorporates a filling-in process is the FACADE (form and color and depth) model proposed by Grossberg and colleagues (Cohen \& Grossberg, 1984; Grossberg, 1987, 1997; Grossberg \& Mingolla, 1985a, 1985b). In this model, a feature contour system (FCS) includes a diffusive filling-in process that computes and distributes brightness and color information across a region, but the filling-in is restricted by signals from a boundary contour system (BCS) that block the filling-in process from spreading into adjacent regions. Subsequent development of the filling-in process has focused on the need for separate black and white filling-in systems (Arrington, 1996; Pessoa, Mingolla, \& Neumann, 1995; Rudd \& Arrington, 2001) and on the need for separate contrast- and luminance-driven systems (Neumann, Pessoa, \& Mingolla, 1998; Pessoa et al., 1995). Across all of these developments, a core idea has been that the filling-in process is based on something like 
diffusion, where brightness or color spreads from edges to surface interiors.

Francis and Rothmayer (2003) used the FACADE model to account for a new type of afterimage involving two images of opposite orientations. On the basis of previous research by Vidyasagar, Buzas, Kisyarday, and Eysel (1999), Francis and Rothmayer found that after viewing two orthogonal bar gratings presented one after the other, observers reported an afterimage similar to the first of the gratings. When two parallel bar gratings were presented, observers tended to report seeing no afterimage at all. When only one bar grating was presented, some observers did report seeing an afterimage of the bar grating, but with less frequency than when two orthogonal bar gratings were presented. The orthogonal orientation of the second bar grating relative to the first seemed critical in the appearance of the afterimage. Francis and Rothmayer referred to the afterimage produced after a sequence of orthogonal patterns as a modal complementary afterimage (MCAI), to reflect its hypothesized relationship to a similar class of amodal orientational complementary afterimages (Hunter, 1915; MacKay, 1957; Pierce, 1900; Purkinje, 1823).

Francis and Rothmayer (2003) used computer simulations of the FACADE model to show that it captured the key aspects of the experimental data. Briefly, the model hypothesizes that the afterimage percepts under these conditions are based on two kinds of afterresponses in the visual system. One kind of afterresponse codes opposite colors (e.g., black/white), whereas the other kind of afterresponse codes opposite orientations (e.g., horizontal/vertical). These afterresponses interact at a neural stage of filling-in, where the color signals spread across surface regions that are defined by orientation signals. Details of the model are discussed below. The properties of the filling-in stage proved critical to the model's ability to account for the afterimage percepts.

In this article, we further explore the properties of the FACADE model in the context of these kinds of afterimages. We describe and test two experimental predictions that follow from the properties of the model. The first prediction describes afterimage percepts that should be seen, using a stimulus that has more than one orientation. The second prediction describes a situation where afterimage percepts should not be seen. Experimental studies show that while the first prediction is valid, the second is not. Finally, we show how the failure of the second prediction forces the model to hypothesize a fillingin mechanism that is not just a diffusive spread of brightness and color information.

\section{Model Description}

FACADE is an extension of a model proposed by Grossberg and Mingolla (1985a, 1985b), who suggested that computational processing in the visual system is divided into a BCS that processes edge, or boundary, information and an FCS that retains information about surface colors and brightness and also provides stages for fillingin of that information to identify the color and brightness of surfaces. The BCS is concerned with identifying the location and orientation of edge-like information. The filling-in stage in the FCS uses the layout of BCS boundary information to define the spread of surface information. A set of connected BCS signals can define a closed region called a filling in domain (FIDO; Grossberg, 1994). The FCS signals that are spatially located within a FIDO are constrained to diffusively spread information only within the FIDO. Separate FIDOs correspond to surface regions with different perceived brightness or color.

Figure 1 shows the key components of FACADE that can account for the properties of MCAIs. Not all connections and interactions are drawn in this schematic representation, and for simplicity the discussion is restricted to achromatic colors. The input image projects to a pixel representation of the black and white components of the image. The opposite color representations compete in a gated dipole circuit (Grossberg, 1972), which creates afterresponses at stimulus offset. A gated dipole

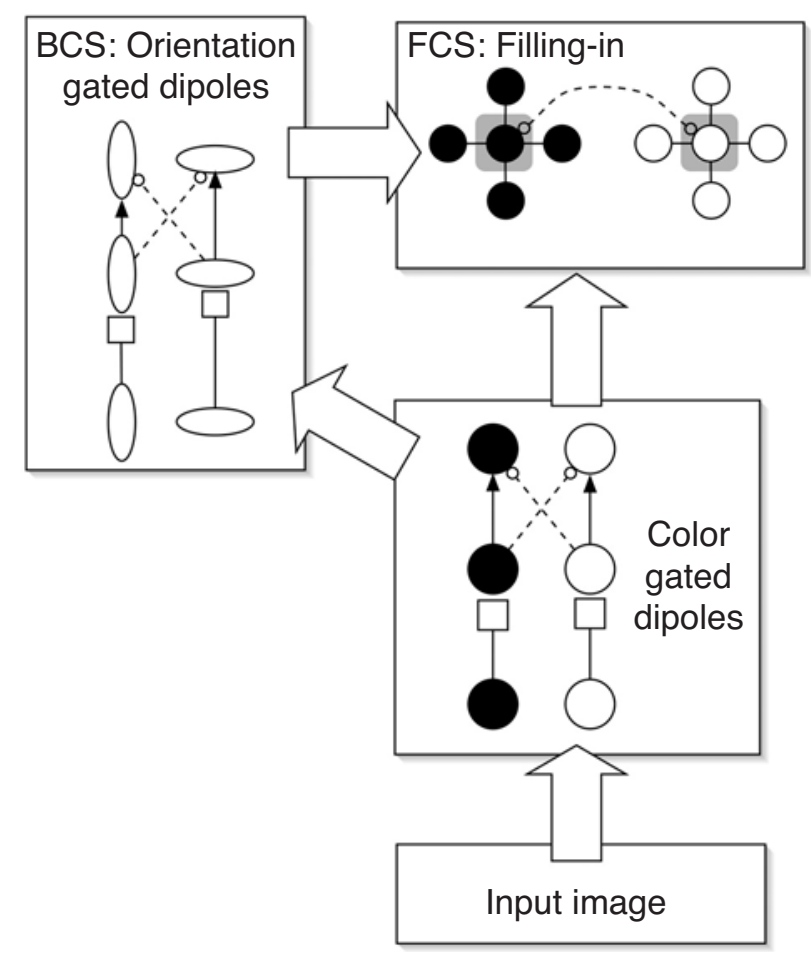

Figure 1. A schematic of the key components of the FACADE theory that are relevant for MCAIs. The input image feeds into a retinotopic representation of black and white, which compete in a gated dipole circuit. The gated dipole circuit produces complementary afterresponses. The black and white information then feeds into edge detection in the BCS, which also contains a gated dipole circuit whose afterresponses code orthogonal orientations. The edges in the BCS guide the spread of black and white information in the FCS filling-in stage to limit the spread of color and brightness information. 
circuit includes parallel channels that compete with each other as signals pass from lower to higher levels of the circuit. Feeding this competition are inputs gated by habituative transmitter gates (schematized as boxes). At stimulus offset, a gated dipole circuit reduces crosschannel inhibition from the stimulated channel to the unstimulated channel. This leads to a transient rebound of activity in the unstimulated pathway. Thus, for a color gated dipole circuit, offset of input to the white channel leads to a brief afterresponse in the black channel, and vice versa.

The color signal at the output of the gated dipole then projects to two different systems: the BCS and the FCS. Cells in the BCS are sensitive to oriented patterns of intensity and correspond to the simple and complex cells of areas V1 and V2 (see Grossberg, Mingolla, \& Ross, 1997, and Raizada \& Grossberg, 2003, for neurophysiological interpretations of the BCS). Within the BCS is another gated dipole circuit that codes orthogonal orientations. Thus, offset of input driving a horizontally tuned cell will lead to an afterresponse in a vertically tuned cell that codes the same retinal position, and vice versa. Other computations, such as excitatory feedback, that group together common orientations, take place in the BCS to insure that boundaries define and segment appropriate regions of an image (Grossberg, \& Mingolla, 1985a, $1985 \mathrm{~b})$. The orientation gated dipole helps to control the duration of persisting responses that are generated by the excitatory feedback loops (Francis, Grossberg, \& Mingolla, 1994) and also contributes to a sharpening of oriented edge detection (Grossberg \& Mingolla, 1985a, 1985b).

Activities from the top level of the color gated dipole and the top level of the orientation gated dipole both feed into the FCS filling-in stage, which contains separate systems for the representation of black and white. The cells in these systems are closely connected so that activity quickly spreads to neighboring cells. As a result, representations of black and white quickly diffuse across cells to fill-in different regions. The diffusion of neural activity is blocked by the presence of appropriately oriented BCS signals. Thus, activity from a horizontally tuned cell in the BCS will prevent black and white activity from spreading up or down for the cells at the corresponding retinal locations in the black and white fillingin systems. In this way, the BCS orientation signals separate different regions, which can then fill-in with different brightness intensities. Reciprocal inhibition between cells in the black and white filling-in systems ensure that a given retinal position can only have either a black or white signal.

Figure 2 shows the development of MCAIs by presenting the different components of the model during a simulated trial. The trial starts with the presentation of a horizontal black and white grating (Figure 2A). The output of the color gated dipole (marked by the black and white circles) shows the input from the horizontal grating. The boundary signals (marked by the oriented ovals) are primarily horizontal. (Black color at a pixel indicates a response from a horizontally tuned cell, and white color at a pixel indicates a response from a vertically tuned cell.) The filling-in stage shows a horizontal grating, and thus a veridical percept. (For simplicity, the black and white filling-in systems are presented together, and middle gray indicates no significant activity in either system.)

Figure 2B shows the model's behavior when the horizontal grating is replaced by a vertical grating. As in the
(A)

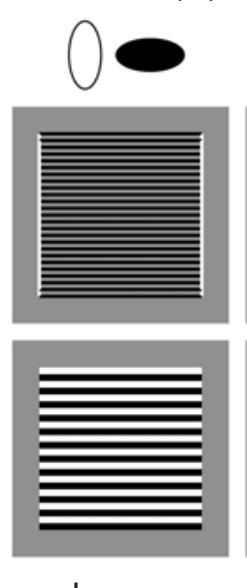

Image
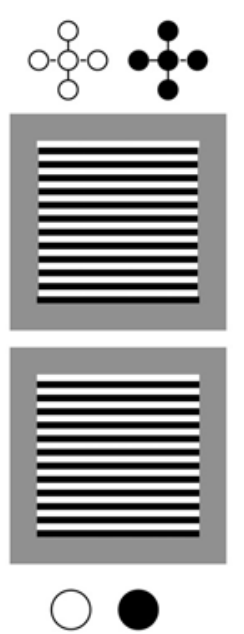

(B)
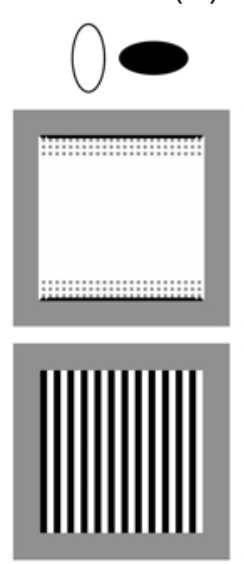

Image
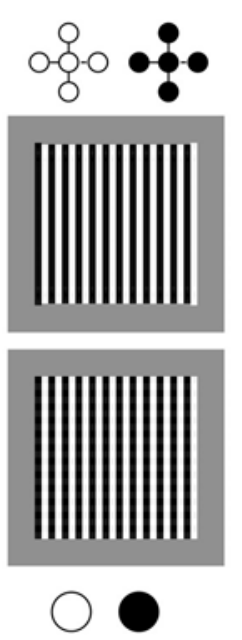

(C)
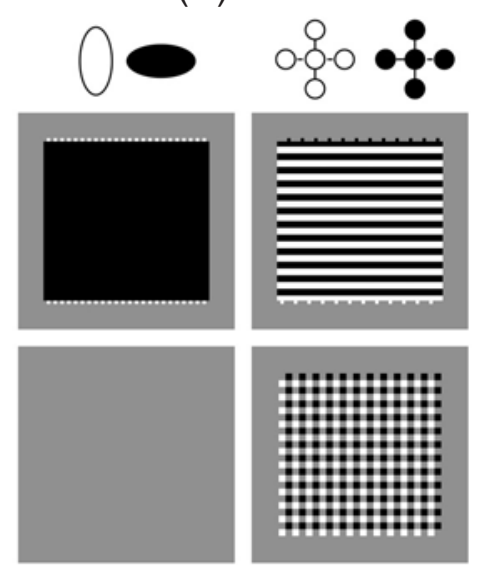

Image
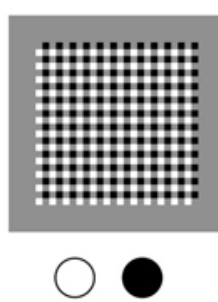

Figure 2. Simulation results for a sequence of images that produce MCAIs. (A) Model behavior during presentation of a horizontal grating. (B) Model behavior during the last of the flickering vertical bar gratings. (C) Model behavior 1 sec after offset of the vertical bar grating. The filling-in stage shows a horizontal pattern, which corresponds to the MCAI. 
experiments of Francis and Rothmayer (2003), this vertical grating flickered with its color complement, and Figure 2B shows the behavior of the model at the end of the last vertical grating. The output of the color gated dipole shows predominantly vertically arranged black and white color signals. However, faintly superimposed on the vertical pattern are black and white horizontal bars. (The faint horizontal stripes may not be visible in the reproduction of the image.) These horizontal stripes are color afterresponses produced by the offset of the horizontal grating. The orientation signals are predominantly vertical (white) because of two effects. First, the presentation of the vertical image produces strong responses among vertically tuned cells at the appropriate positions on the edges of the bars. Second, the offset of the horizontal grating causes rebounds in the orientation gated dipole that produce strong vertical boundary responses. After excitatory feedback groups together cells with similar orientations, a dense block of vertically tuned cells respond across the entire grating. The filling-in stage shows a vertical grating, which corresponds to a veridical percept.

Figure 2C shows the model's behavior $1 \mathrm{sec}$ after the offset of the vertical grating. The input image is a blank gray background. The responses of the color gated dipoles produce a checkerboard pattern, which is due to afterresponses generated by both the vertical and horizontal bar gratings. The orientation signals are primarily horizontal because offset of the vertical bar grating produces afterresponses among horizontally tuned cells. In this simulation (and all the simulations reported here), the gated dipole for color operates with a slower time constant than does the gated dipole for orientation, which means that the color afterresponses fade more slowly than do the orientation afterresponses. Thus, the activities across the color gated dipoles are a combination of both inducing stimuli, but the activities across the orientation gated dipoles are almost entirely determined by the second stimulus only.

The filling-in stage in Figure 2C shows a horizontal bar grating, which corresponds to the MCAI percept. The filling-in stage produces this pattern because the horizontal boundary signals constrain the filling-in signals to spread only left and right, but not up and down. Thus, the dark and light columns of inputs from the color gated dipole spread across each other and cancel out. On the other hand, the dark and light rows in the color gated dipole are kept separate and so support activity at the filling-in stage. The net effect is that the orientation afterresponses force the filling-in stages to "pick out" the horizontal pattern in the outputs of the color gated dipoles. This simulation was used by Francis and Rothmayer (2003) to demonstrate that the model produces the primary effect noted by Vidyasagar et al. (1999). Other simulations showed that the model did not produce an MCAI if the second frame contained a bar pattern of the same orientation as the first or if it was blank.
FACADE's explanation of these afterimage percepts is similar, in some respects, to the explanation proposed by Vidyasagar et al. (1999). As in FACADE theory, they proposed that cross-orientation inhibition and a fading trace were critical to the development of afterimage percepts. However, they did not consider the importance of distinguishing between boundary and surface mechanisms or the critical role that filling-in mechanisms play in accounting for the appearance of MCAIs. As a result, their explanation is unclear about what kinds of afterimage percepts should be produced under new circumstances. As described in the following sections, FACADE makes very precise and testable predictions about MCAIs with new stimuli.

\section{EXPERIMENT 1 Predicted MCAIs With Different Stimulus Types}

The explanation of MCAIs proposed by the FACADE model suggests that the afterimage percept is not just an amplified afterimage of the first grating. Rather, the MCAI is a construction that depends critically on having appropriate color afterresponses and orientation afterresponses that interact in a filling-in process. This fillingin process can cause strong afterresponses to wash out, even as it supports the visibility of weak afterresponses. As such, the theory predicts that MCAIs might appear that look quite different from the first inducing stimulus.

Figure 3 shows simulation results that demonstrate one example of this prediction. The first inducing pattern (Figure 3A) is a black grid on a gray background. The orientation-sensitive cells of the BCS enclose the black grid, and the filling-in stage is a veridical representation of the stimulus. When the grid is replaced by a flickering vertical black and white bar grating, as in Figure $3 \mathrm{~B}$, the orientation-sensitive cells of the BCS include vertical responses generated by the vertical bar grating and vertical afterresponses from the horizontal components of the previously shown grid. A flickering grating was used because it tends to reduce (but not eliminate) the color-complement afterresponses at the offset of the grating. Most of the horizontal afterresponses from the vertical components of the grid are washed out by the presence of the strong vertical contours in the bar grating.

Figure 3C shows the model's behavior shortly after offset of the vertical black and white bar grating. The color gated dipoles show a pattern of white squares. These afterresponses occur because of the overlap of parts of the first inducing stimulus (the grid) and the second inducing stimulus (the bar grating). Where both the grid and the grating were black, there is a bit more habituation than elsewhere, which leads to the pattern of squares. The orientation-sensitive cells of the BCS exhibit strong horizontal activity, due to afterresponses generated at the offset of the vertical bar grating. At the filling-in stage, the horizontal boundary signals allow the squares of white color activity to spread horizontally 
(A)

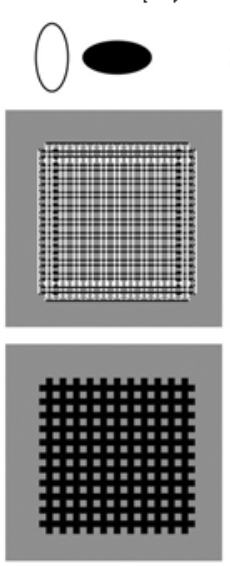

Image
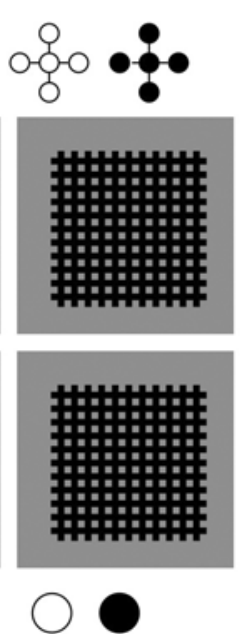

(B)
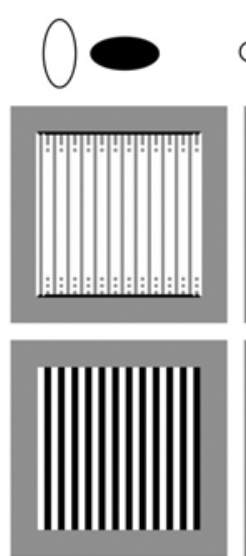

Image
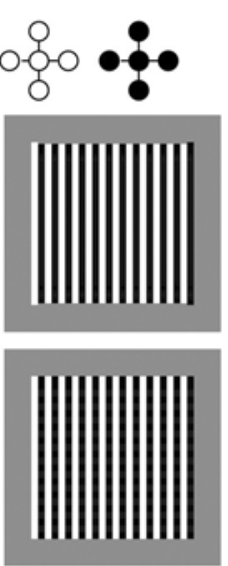

$\bigcirc$
(C)
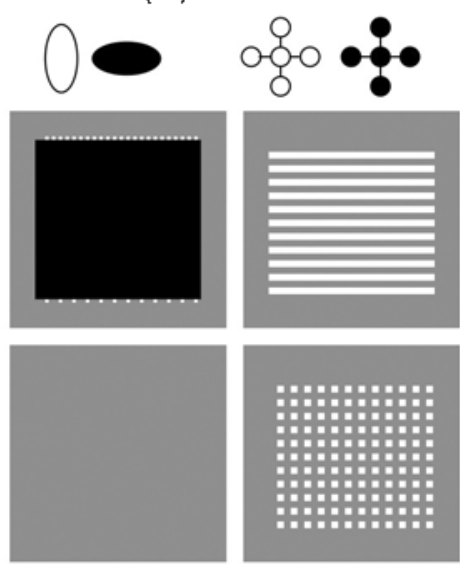

Image
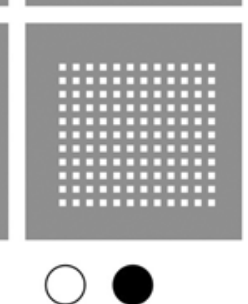

Figure 3. Simulation results for a sequence of images when the first image includes a black grid. (A) Model behavior during presentation of a black grid. (B) Model behavior during the last of the flickering vertical bar gratings. (C) Model behavior 1 sec after offset of the vertical bar grating. The filling-in stage shows a horizontal pattern, which corresponds to the MCAI.

but separate the vertical gaps between the squares. The net result is that the filling-in stage produces a horizontal bar grating pattern. Experiment 1 tests this model prediction.

\section{Method}

All stimuli were created and presented with MATLAB, using the Psychophysics Toolbox extensions (Brainard, 1997; Pelli, 1997), on a PC running Windows 98 with a monitor that refreshed at $75 \mathrm{~Hz}$.

Figure 4 schematizes one of the trials. The first stimulus was always a grid made of black $\left(0.6 \mathrm{~cd} / \mathrm{m}^{2}\right)$ horizontal and vertical bars (as shown in Figure 4$)$ on a gray $\left(24 \mathrm{~cd} / \mathrm{m}^{2}\right)$ background. The grid was shown for $1.5 \mathrm{sec}$ and then replaced by a second stimulus. The second stimulus was either a vertical grating, a horizontal grating, or a blank image (which showed the gray background). If the second stimulus was a grating, it flickered by alternating the black and white $\left(128 \mathrm{~cd} / \mathrm{m}^{2}\right)$ bars for durations of $100 \mathrm{msec}$ each. The second stimulus was presented for a total of $1.5 \mathrm{sec}$. After the second stimulus, the screen showed only the gray background for $1.5 \mathrm{sec}$. This was then replaced by a box of random dots, which was a cue for the observer to report any afterimage that was seen just before the random dots appeared. The observers were advised that if the afterimage percept changed during the blank interval, they were to report the properties of what was visible just before the random dots appeared.

The grid consisted of nine vertical and nine horizontal black bars, with equally spaced gaps that showed the gray background. At a viewing distance of $60 \mathrm{~cm}$, the maximum extent of the grid was approximately $10^{\circ}$ visual angle in height and width. The vertical and horizontal gratings and the box of random dots were the same size.

The observers were instructed to describe any afterimage by selecting from four possibilities: vertical grating, horizontal grating, grid, and nothing. They entered their responses on a keyboard. Once an observer picked a response, another keypress started the next trial. To minimize interactions across trials, a forced delay of $15 \mathrm{sec}$ was introduced before the start of the next trial.

Fifteen replications for each of the three second stimulus types were randomly mixed in an experimental session of 45 trials. Eight naive observers were recruited from the experimental subject pool at Purdue University. They received course credit for participation in the experiment. Each observer was run separately in a room that used regular overhead lighting.

\section{Results}

Figure 5 shows the proportion of different responses for each of the second frame conditions. Each graph is based on data from 120 trials.

Figure 5A shows the proportion of reports when the second frame was blank. The reports are divided between seeing nothing and seeing an afterimage whose shape was similar to the inducing grid pattern. No observers reported seeing a horizontal or vertical bar grating.

Figure 5B shows the proportion of reports when the second stimulus was a horizontal bar grating. Consistent with the model's prediction, reports of a vertical afterimage are more common than anything else. Likewise, Figure $5 \mathrm{C}$ shows the proportion of reports when the second stimulus was a vertical bar grating. Reports of a horizontal bar grating afterimage were more common than anything else.

\section{Discussion}

The experimental data show that MCAIs need not have the same shape as the first inducing stimulus. This finding validates the model's prediction that these kinds of afterimages are the result of interactions between orientation afterresponses, color afterresponses, and a fillingin process. Thus, we find confirmatory evidence for the explanation of MCAIs proposed by Francis and Rothmayer (2003). Namely, the data are consistent with the FACADE model and its hypothesized afterresponses.

\section{EXPERIMENT 2 Predicted Absence of MCAIs}

We next used the FACADE model to predict types of inducing stimuli that should not demonstrate an MCAI. When the color gated dipole afterresponses within an 


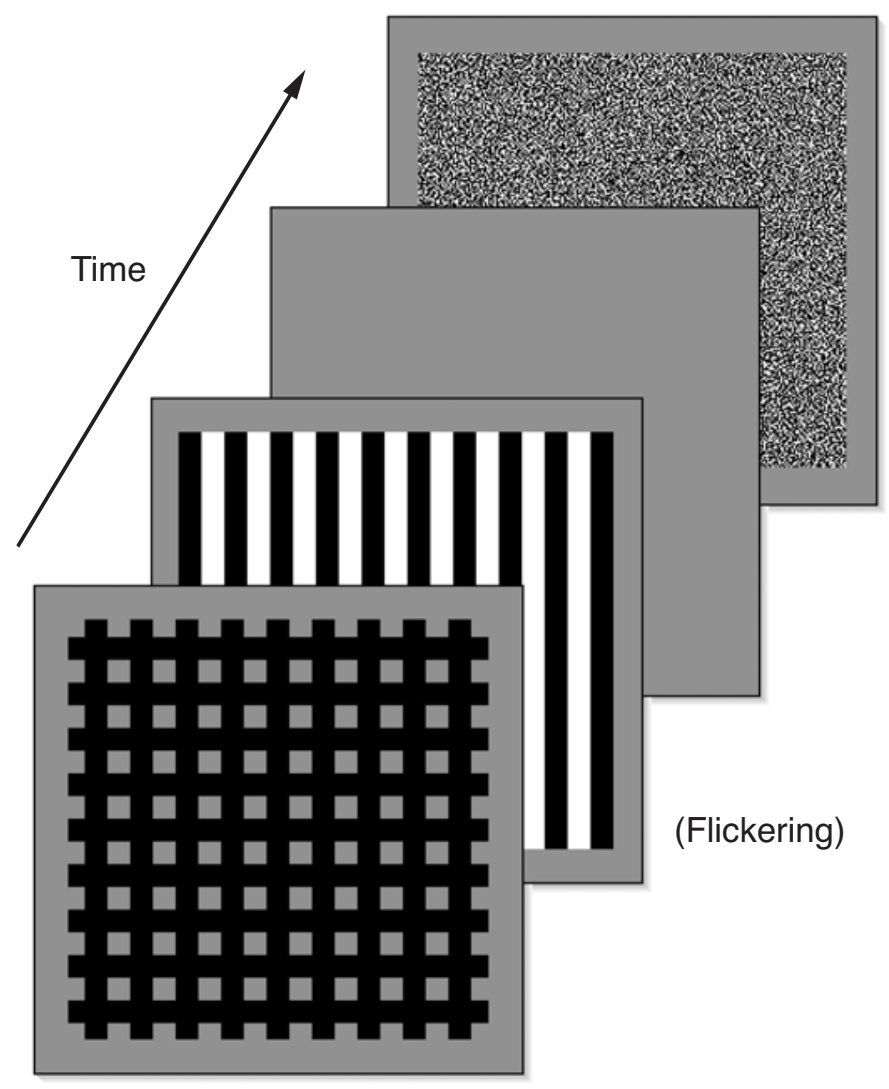

Figure 4. The sequence of frames during a trial of Experiment 1. The observer's task was to report on any seen afterimages just before the box of random dots appeared.

FIDO are balanced between black and white signals, they should cancel each other out, giving rise to a percept of the gray background. Figure 6 shows one example of this situation.

The first inducing grating (Figure 6A) contains horizontal bars that switch their polarity at midsection. In this split grating, each horizontal bar has a black and a white side to it. The model's representation at the fillingin stage is essentially veridical. The second inducing grating (Figure 6B) is a flickering vertical black and white bar grating. Although the color gated dipole includes some afterresponses from the first grating, the orientation signals are predominantly vertical, and the filling-in stage veridically reflects the vertical grating.

Shortly after offset of the vertical grating (Figure 6C), the color gated dipoles show a mix of afterresponses from both inducing gratings. Offset of the vertical signals in the $\mathrm{BCS}$ produces afterresponses that are predominantly horizontal. As in Figure 3, these afterresponses are almost entirely determined by the orientation of the second grating, although there may be a slight strengthening of the horizontal afterresponses due to the presence of the vertical contour in the middle of the split grating. At the filling-in stage, the horizontal orientation signals force the color input to spread left and right. However, since there are equal amounts of black and white within each horizontal row, the signals cancel each other out (except at the top and bottom of the grating where small effects occur, these will disappear in a more sophisticated simulation). The net result is that the model simulation predicts that observers should not see an MCAI when the first inducer is a split grating, even though the two inducing gratings are largely orthogonal to each other.

The simulation is similar even if the black and white afterresponses are not exactly balanced. In such a situation, the average color within each row is diffused across the entire row. Thus, the filling-in stage will produce a square that is slightly brighter or darker than the background. Thus, with a diffusive filling-in stage, the model must predict that a split grating will not produce a visible MCAI.

\section{Method}

Conditions were similar to those of Experiment 1, and Figure 7 schematizes one of the trials. The first inducing grating could be a split horizontal grating (as shown in Figure 7), a split vertical grating, a continuous horizontal grating, or a continuous vertical grating. The second grating could be a vertical grating, a horizontal grating, or blank.

The observers were instructed to describe the afterimage by selecting from five possibilities: continuous horizontal grating, con- 

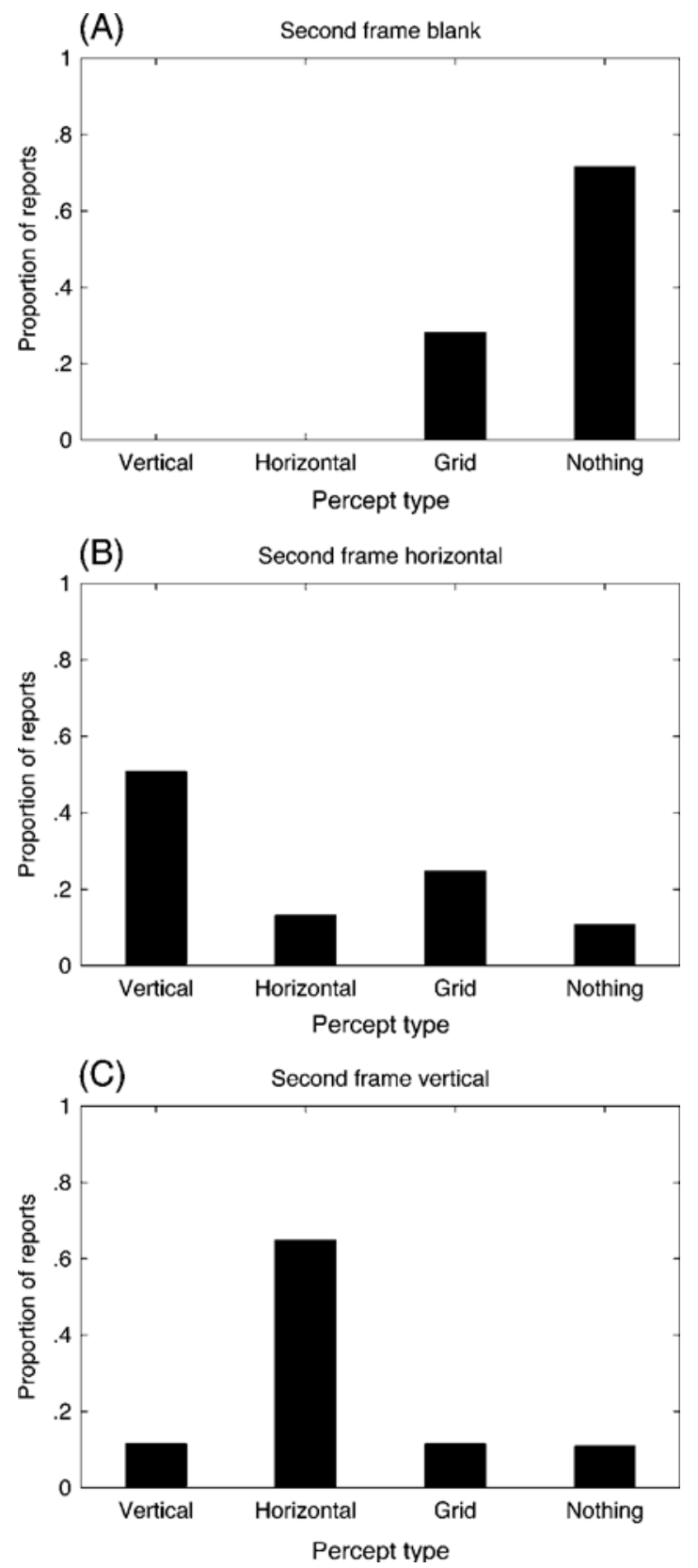

Figure 5. Results of Experiment 1. The data are divided according to the orientation of the two gratings. (A) When the second frame was blank, observers reported seeing either an afterimage shaped like the grid or not seeing any afterimage at all. (B) When the second frame was a horizontal bar grating, observers tended to report seeing a vertical grating as an afterimage. (C) When the second frame was a vertical bar grating, observers tended to report seeing a horizontal grating as an afterimage.

tinuous vertical grating, split horizontal grating, split vertical grating, and nothing. They entered their responses on a keyboard. Once an observer picked a response, another keypress started the next trial. Before the next trial started, there was a forced delay of $10 \mathrm{sec}$.

Five replications of each combination of the four first stimulus types and three second stimulus types were randomly mixed in an experimental session of 60 trials. Eleven naive observers were re- cruited from the experimental subject pool at Purdue University. The observers received course credit for participation in the experiment.

\section{Results}

In the analysis of the data, we grouped the reports according to whether the observer indicated seeing an afterimage that was parallel or orthogonal to the first stimulus. Figure 8 shows results when the first stimulus was a continuous bar grating and the second stimulus was either blank (Figure 8A), parallel to the first grating (Figure $8 \mathrm{~B}$ ), or orthogonal to the first grating (Figure $8 \mathrm{C}$ ). The results are essentially a replication and generalization of the results in Francis and Rothmayer (2003). Each graph plots the proportion of responses that correspond to the different afterimage percepts, and each is based on data from 110 trials.

Figure 8A shows the proportion of reports when the second stimulus was blank. The reports are largely divided between seeing nothing and seeing an afterimage of a continuous bar grating parallel to the inducing grating. Presumably, the afterimage of a continuous bar grating is a retinal or color-based afterimage. Francis and Rothmayer (2003) found similar results and showed how they corresponded to different parameter settings in the FACADE model.

Figure $8 \mathrm{~B}$ shows the proportion of reports when the second stimulus was parallel to the first stimulus. The majority of reports are of seeing nothing, which is consistent with the findings of Francis and Rothmayer (2003) and with the model.

Figure $8 \mathrm{C}$ shows the proportion of reports when the second stimulus was orthogonal to the first stimulus. Almost all reports were of an afterimage that was a continuous bar grating parallel to the first inducing grating. Most notably, reports of the afterimage were higher when the second image was an orthogonal grating than when the second image was blank. This result is also consistent with the observations of Vidyasagar et al. (1999), Francis and Rothmayer (2003), and the model (see Figure 2).

Thus, the results shown in Figure 8 replicate the findings of Francis and Rothmayer (2003) and demonstrate that under the proper conditions, observers in this experiment did see MCAIs. With this established, it is appropriate to explore the model's predicted absence of MCAI percepts when the first grating is split. Figure 9 shows results when the first stimulus was a split bar grating and the second stimulus was either blank (Figure 9A), parallel to the first grating (Figure 9B), or orthogonal to the first grating (Figure 9C).

Figure 9A shows the proportion of reports when the second stimulus was blank. The reports are divided between seeing nothing and seeing an afterimage of a split bar grating parallel to the inducing grating. Similar to the continuous inducing bar grating, the afterimage here is probably a retinal or color-based afterimage. Francis and Rothmayer (2003) noted that there were differences across observers such that some almost always reported seeing a color afterimage, whereas others almost never reported seeing a color afterimage. Figure 10 shows that 
(A)

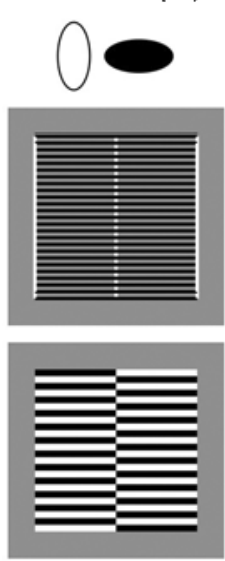

Image
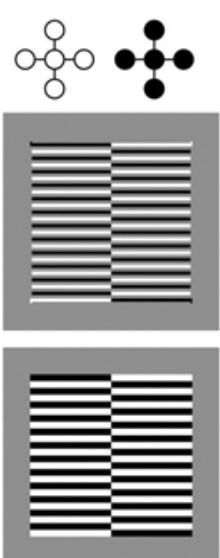

$\bigcirc 0$
(B)
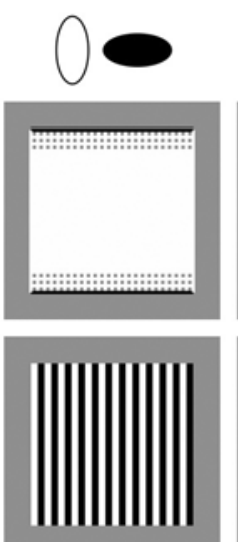

Image
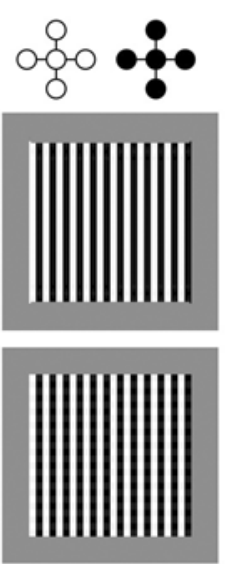

$\bigcirc$
(C)
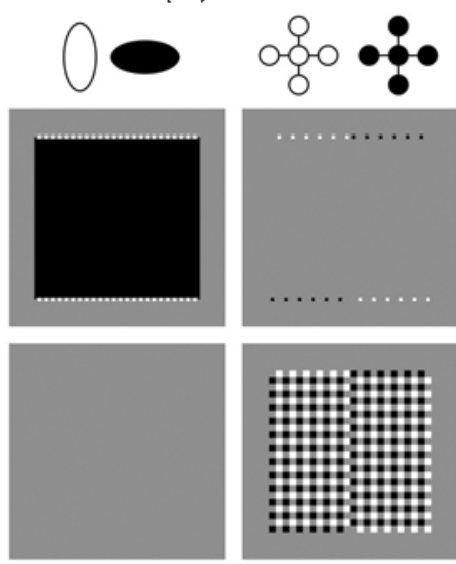

Image
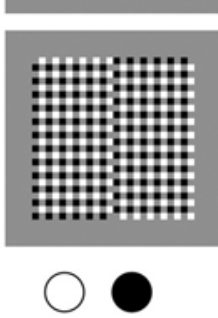

Figure 6. Simulation results for a sequence of images when the first image includes a split grating. (A) Model behavior during presentation of a split horizontal grating. (B) Model behavior during the last of the flickering vertical bar gratings. (C) Model behavior 1sec after offset of the vertical bar grating. The black and white afterresponses spread horizontally and cancel each other out, so that only the gray background is visible.

the same trend is present in our data when the second stimulus was blank. Each point in Figure 10 corresponds to a separate observer, and the plot compares the percentage of nothing responses to the percentage of re- sponses that were the same shape as the first inducer grating. There seems to be a continuum of observer types. Some observers (lower right corner) almost always report seeing a retinal or color-complement afterimage,

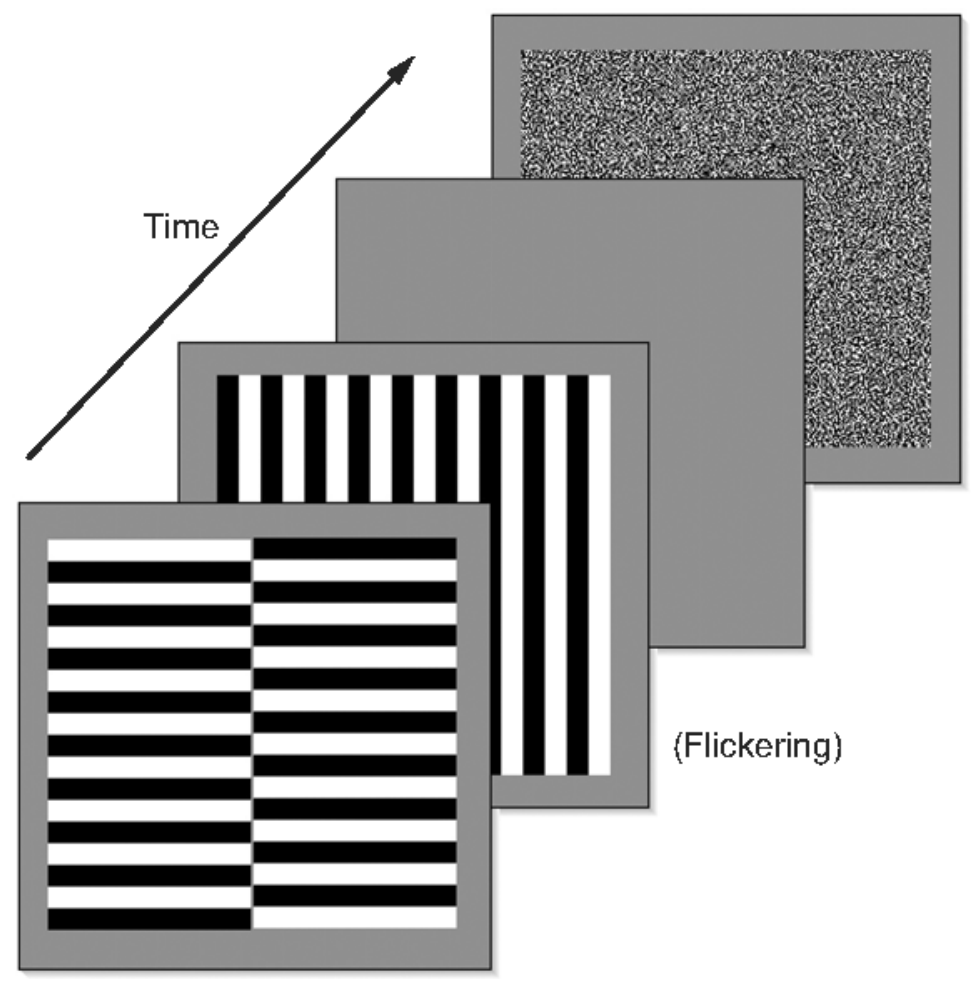

Figure 7. The sequence of frames during a trial of Experiment 2. The observer's task was to report on any seen afterimages just before the box of random dots appeared. 


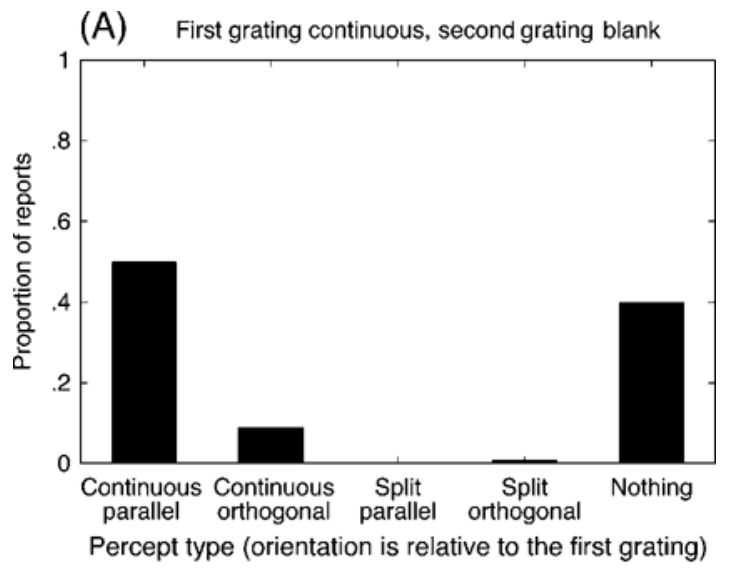

(B) First grating continuous, second grating parallel

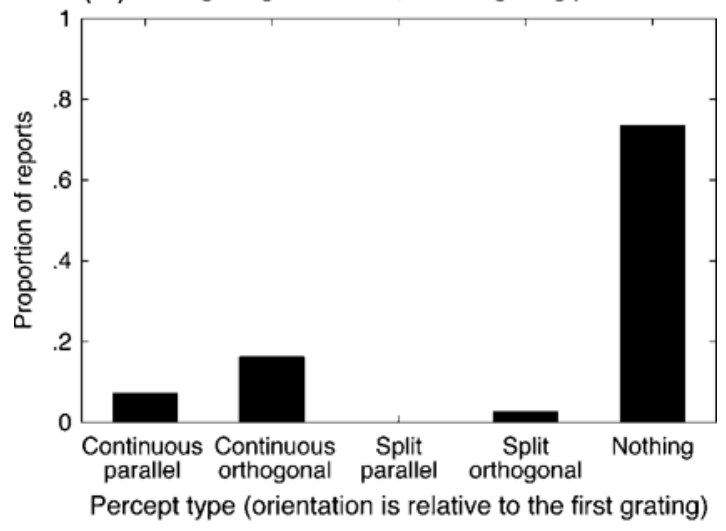

(C) First grating continuous, second grating orthogonal

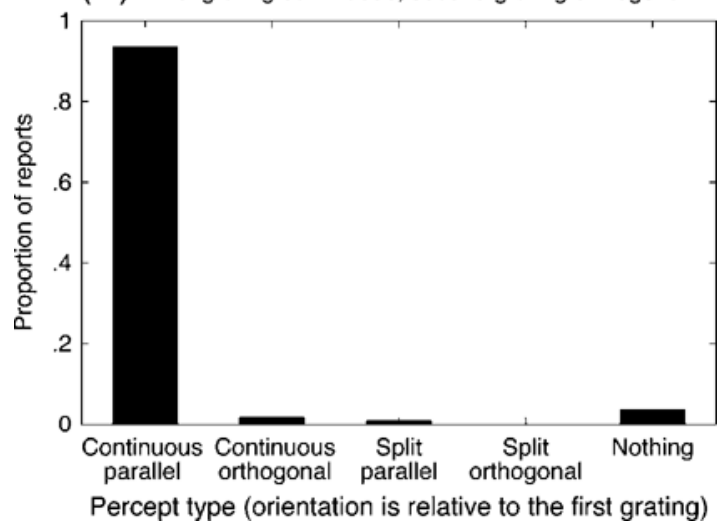

Figure 8. Results of Experiment 2 when the first frame contained a continuous horizontal or vertical grating. The data are divided according to the orientation of the two gratings. (A) When the second frame was blank, observers reported seeing either no afterimage or an afterimage of a continuous grating with an orientation parallel to the first grating. (B) When the second frame was a bar grating that was parallel to the first grating, observers tended to report seeing no afterimage. (C) When the second frame was a bar grating that was orthogonal to the first grating, observers reported seeing an afterimage of a continuous grating with an orientation parallel to the first grating. This percept is the MCAI. whereas others (upper left) almost never report such afterimages. There are also observers with response frequencies in between. Thus, the percentages in Figures 8A and 9A show substantial variability across observers.

Figure 9B shows the proportion of reports when the second stimulus was parallel to the first stimulus. The majority of reports are of seeing nothing. This result is consistent with the findings of Francis and Rothmayer (2003) and with the model.

The data that test the model's prediction are in Figure $9 \mathrm{C}$, which shows the proportion of reports when the second stimulus was orthogonal to the first stimulus. The model predicts that the predominant percept should be of nothing. Contrary to this prediction, almost all reports were of an afterimage that was a split bar grating parallel to the first inducing grating.

\section{Discussion}

On trials with continuous bar gratings for the first stimulus, observers saw an MCAI when the second stimulus was orthogonal to the first. When the second stimulus was blank, we find that individual differences can have a large impact on whether afterimages are seen. All of these data replicate the findings of Francis and Rothmayer (2003) and are consistent with the properties of the FACADE theory. However, contrary to the model's prediction, the split grating produced an MCAI that also contained a split. The model predicted that the opposite colors of the split would spread across each other and thus cancel each other out.

The experimental results suggest that for the FACADE theory to account for the properties of these afterimages, the filling-in mechanism must be different than is presently hypothesized. In the next section, we describe a mechanism that is consistent with previously hypothesized properties of filling-in but is also able to account for the new properties of MCAIs.

\section{A NEW FILLING-IN MECHANISM}

Previous versions of the FACADE model have hypothesized that the spreading of FCS information at the filling-in stage was similar to a diffusive process, like the distribution of heat in a room. However, this type of filling-in mechanism cannot account for the existence of MCAIs with a split grating. At the offset of a vertical grating in an MCAI inducing sequence, BCS-defined FIDOs consist of individual horizontal rows, and a diffusive process should spread white and black signals evenly across each row. Since equal amounts of white and black are present on each side of the split grating (and its color afterresponses), the black and white signals should cancel each other out as they diffuse horizontally, as in Figure 6. However, this is not the percept.

As we looked for an alternative filling-in mechanism, we wanted to be sure that the new mechanism would also 


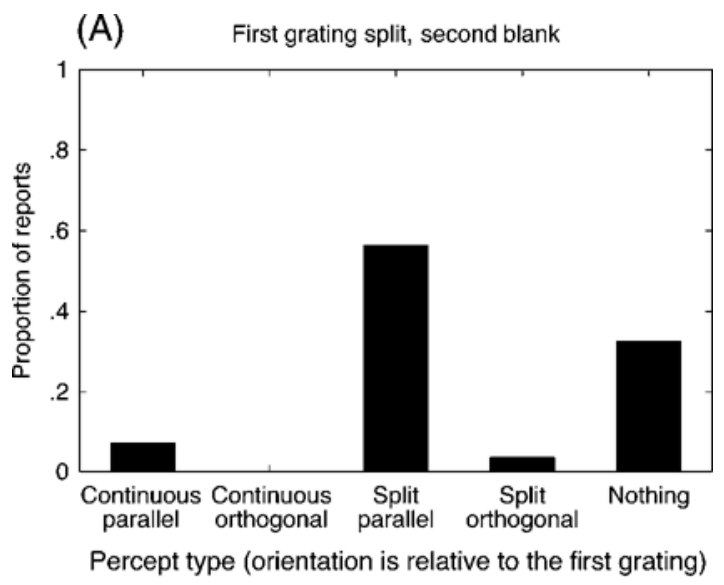

(B)

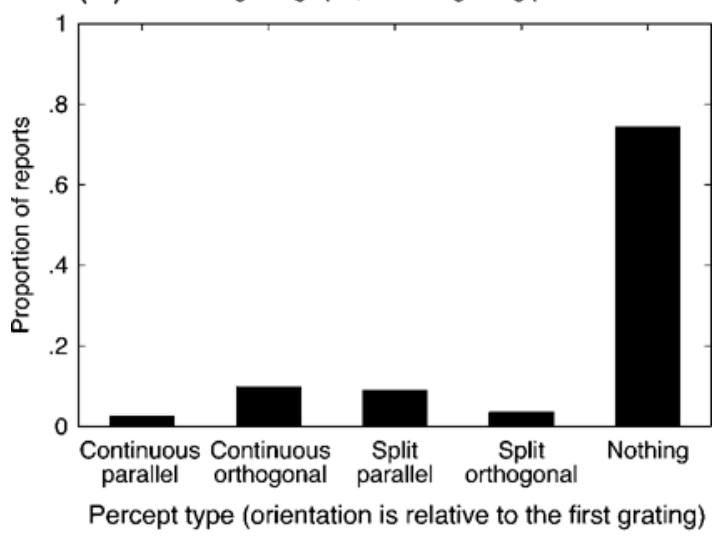

(C) First grating split, second grating orthogonal

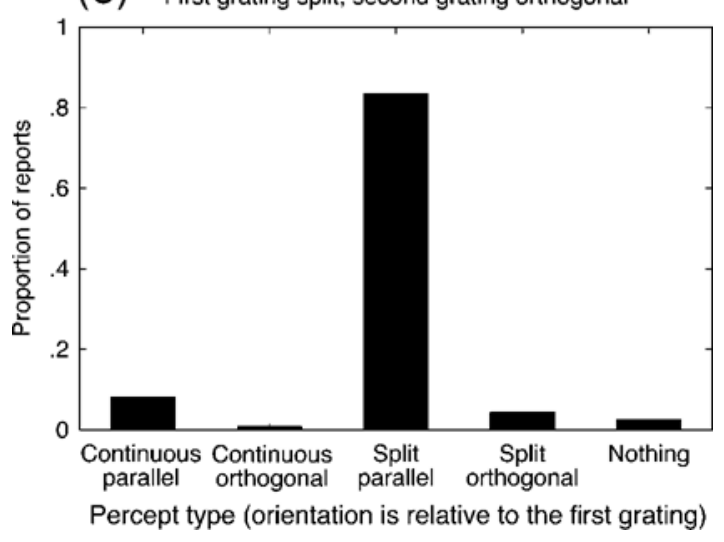

Figure 9. Results of Experiment 2 when the first frame contained a split horizontal or vertical grating. The data are divided according to the properties of the second frame. (A) When the second frame was blank, observers reported seeing either no afterimage or an afterimage of a split grating with an orientation parallel to the first grating. (B) When the second frame was a bar grating that was parallel to the first grating, observers tended to report seeing no afterimage. $(C)$ When the second frame was a bar grating that was orthogonal to the first grating, observers reported seeing an afterimage of a split grating with an orientation parallel to the first grating. The last result is inconsistent with the model's prediction that observers should see no afterimage under this condition. work in the many other situations where filling-in has been proposed. To account for phenomena like neon color spreading and the watercolor illusion (da Pos \& Bressan, 2003; Pinna et al., 2001), filling-in must be capable of spreading across a fairly large region of visual space. The FACADE theory also uses this property to ensure proper computation of surfaces in depth (Grossberg, 1997). To account for these phenomena, the filling-in mechanism cannot be fundamentally limited in spatial extent, such as might exist from feedforward connections from one neural layer to another.

Many alternative filling-in mechanisms either failed to produce a split grating MCAI or the standard continuous grating MCAI. Our analysis suggests that whatever filling-in mechanism is involved in the MCAI percepts, the presence of boundary signals must force it to be an interpolating process rather than an extrapolating process. On the other hand, the role of filling-in in the FACADE theory requires it to be an extrapolating process when appropriate boundaries are not present. Thus, for the filling-in process in FACADE to account for our data, we must revise the nature of the interaction between boundaries and filling-in, and we must also revise the nature of how surface information spreads. We expect that a variety of systems can meet the necessary requirements, and we outline one such system below. Simulations demonstrate the new filling-in mechanism's ability to account for the properties of MCAIs. A mathematical description of the simulations is provided in the Appendix.

Figure 11 schematizes the proposed filling-in mechanism. Each cell in a filling-in stage is hypothesized to have a receptive field that samples activities from other cells in the filling-in stage. Part of this receptive field is schematized in Figure 11A, which shows its flower shape. The receptive field contains individual subunits that form the petals. Each petal of the receptive field samples other cells in the surface representation along a particular line drawn outward from the location of the sampling cell.

The behavior of one of these subunits is schematized in Figure 11B. The white circles on the bottom indicate a row of cells in the white filling-in stage. The black circles indicate a row of cells in the black filling-in stage at the same retinal locations. The activities of these cells correspond to the perceived brightness of a position in visual space. The schematized subunit extends leftward from the center of the receptive field and samples activities from other cells in the brightness stage. The sampling of cells is biased in two ways. First, the presence of a BCS boundary signal of an orientation different from the direction of sampling (in this case, horizontal) can block the sampling process from extending beyond that boundary. The lines at different pixel locations in the subunit receptive field schematize which boundary orientations will block sampling. For example, a vertical boundary at the third location from the right would block sampling from that cell and from cells that are in more 


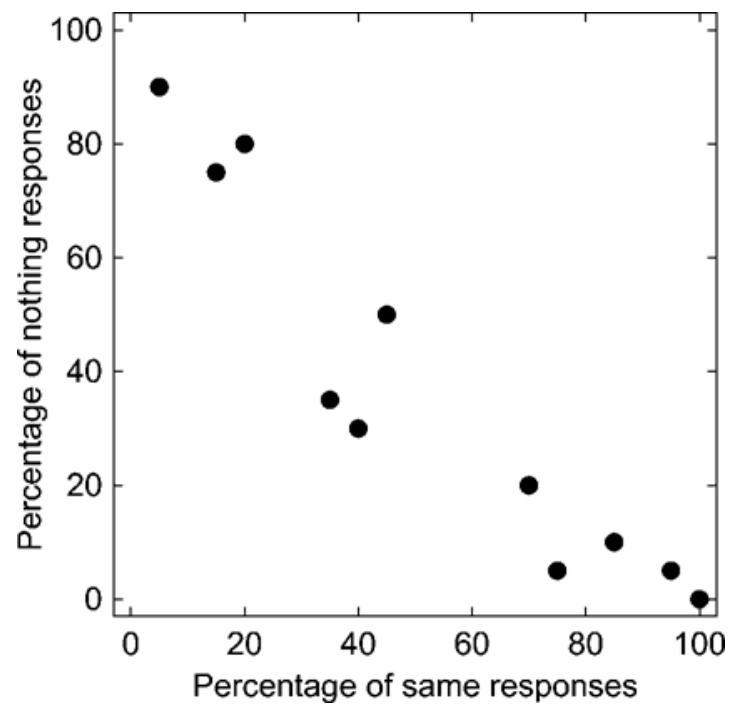

Figure 10. Differences across observers in Experiment 2 when the second frame was blank. Each point corresponds to the responses of 1 observer. The $x$-axis indicates the percentage of times an observer reported an afterimage with a shape similar to the first frame (possibly a retinal afterimage). The $y$-axis indicates the percentage of times an observer reported seeing no afterimage.

distant locations in this subunit. Second, the receptive field only samples as far out as there is an active value to sample. It will sample the activities of any cells between the furthest active cell to the center of the receptive field, whether those intermediate cells are active or not. However, it will not sample inactive cells that are beyond the furthest active cell. These restrictions on sampling of cells prevent the subunit from inappropriately sampling from other surfaces.

Each petal of the receptive field functions in a similar way, sampling from cells in a straight line away from the receptive field center. Each subunit is blocked from sampling cells that lie beyond a BCS boundary signal of an orientation different from the direction of sampling. Each subunit also only samples out to the most distant active sampled cell. In addition, the center of the receptive field samples from itself, if it is active. The center of the receptive field is an additional subunit of the receptive field.

Each sample of a cell's activity includes both excitatory input from the cell in the same filling-in stage (for example, white) and inhibitory input from the opposing filling-in stage (black) at the same pixel location. The inhibition is schematized in Figure 11B as the dashed lines drawn from the black cells to the white receptive field subunit.

If at least two subunits of the receptive field are active, a signal is fed back to the sampling cell. A subunit is active if it samples from at least one active cell in the filling-in stage. This feedback process allows for further changes in activity among other cells and eventually reaches an equilibrium state.
The advantage of this scheme over the diffusion approach to filling-in is twofold. First, it acts essentially the same as the diffusive approach for many situations, including those where the spread of brightness or color information has been used to explain a variety of percepts that have been addressed by FACADE, including neon color spreading (Grossberg \& Mingolla, 1985a), brightness percepts (Grossberg \& Kelly, 1999; Grossberg \& Todorović, 1988), 3-D vision (Grossberg \& McLoughlin, 1997), and aspects of figure-ground segmentation (Grossberg, 1994, 1997). Without blocking boundary signals, two petals of the receptive field can sample from neighboring positions of the same color and become active, thereby spreading the color information from an edge to the interior. Such information will then extrapolate until it is constrained by BCS boundaries. This property is critical for those explanations.

Second, in the special case of CAIs, the dense arrangement of boundary signals will allow brightness signals to interpolate inward but will preclude them from spreading outward. For example, at offset of a vertical grating in the second frame of the MCAI sequence, the boundary afterresponses (after completion by the BCS) are a

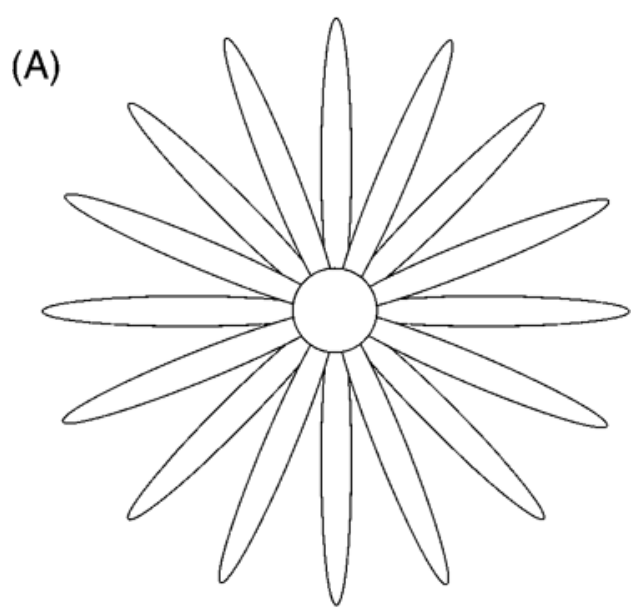

(B)

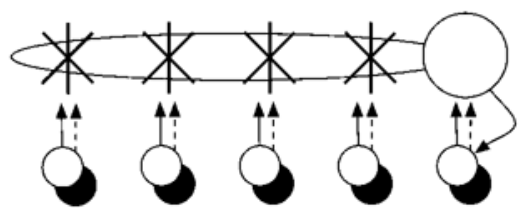

Figure 11. Proposed receptive fields of cells in the filling-in planes. (A) Each cell has a flower-shaped receptive field. Each petal acts as an independent subunit. The cell receives feedback if at least two of the subunits are activated. (B) Details of one petal for a cell in the white filling-in plane. The petal stretches horizontally to the left of the center of the receptive field. At each location, it samples excitatory input from other cells in the white filling-in plane and samples inhibitory input from corresponding cells in the black filling-in plane. In addition, BCS boundary signals that are different from the orientation of the petal can block sampling from locations beyond the boundary. 
dense set of horizontal boundaries. As a result, each row consists of its own FIDO. Since each FIDO is a singlepixel row, there are only two ways to activate the two subunits of a target cell's receptive field. One is to have active cells of the same color on either side of the target cell's receptive field. The other way is, if the target cell is itself active, to have at least one other cell of the same color active on either side of the target cell's receptive field. Thus, in the context of CAIs the filling-in mechanism allows for interpolation of brightness but does not allow for extrapolation. For the stimuli used by Francis and Rothmayer (2003) and in Experiment 1, this interpolation mechanism acts much like a diffusive filling-in mechanism because the black/white signals can interpolate over blank spaces in between. The interpolation characteristic is more significant for an explanation of the MCAI for the split grating.

Figure 12 shows the model's behavior when the first stimulus is a split grating. When the gratings are present (Figures 12A and 12B), the activities across the fillingin stage are veridical representations of the gratings. Offset of the vertical grating produces the desired split horizontal MCAI. To understand why the model matches the percept, consider the checkerboard pattern produced at the color gated dipole output. The white checks on the left and right sides are vertically out of phase because the phase shift of the split horizontal inducing grating. With the proposed filling-in procedure, the white checks fill-in the horizontal space between them but cannot spread to the opposite side, where every signal is black or middle gray (which indicates neither black nor white). The same is true for corresponding black checks, so the black and white signals are kept separate and do not cancel each other out.
Additional simulations of FACADE with the new fillingin mechanism show that it behaves equivalently to the diffusive filling-in process that was used by Francis and Rothmayer (2003) to create Figures 2 and 3. Although there are quantitative differences, the spatial pattern of activities in the filling-in stage matches the patterns in earlier simulations.

Significantly, Hong and Grossberg (2003) also proposed a filling-in mechanism utilizing long-range receptive fields. Their motivation was that simulations of the diffusive filling-in process were computationally slow (because information travels only to the nearest neighbors). They showed that their alternative mechanism could be integrated into the FACADE theory to allow for a notable number of brightness phenomena. Thus, there is both experimental and computational support for a need to deviate from the traditional diffusive filling-in process.

Finally, the proposed mechanism generally agrees with the neurophysiological finding (Friedman et al., 2003) that color-sensitive cells are also orientation sensitive. The separate lobes of the proposed filling-in cells are very orientation and color selective, and the cells identified by Friedman et al. may be neurophysiological instantiations of the proposed mechanism. Of course, more neurophysiological and modeling research is needed to determine whether the proposed mechanism exists in the underlying neurophysiology.

\section{CONCLUSIONS}

We elaborated on the observations of Vidyasagar et al. (1999) and Francis and Rothmayer (2003) to investigate properties of filling-in mechanisms. The first experi-
(A)

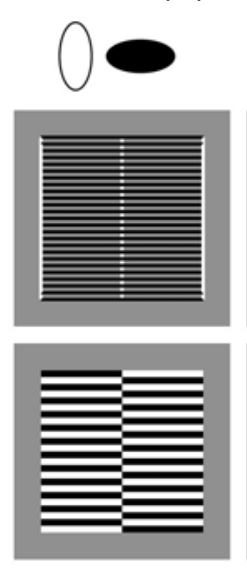

Image
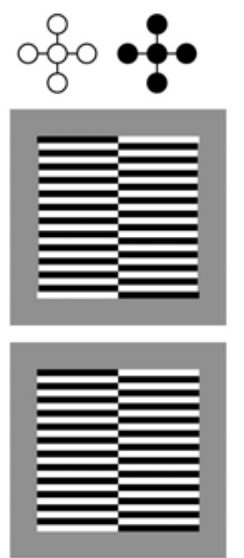

$\bigcirc$
(B)
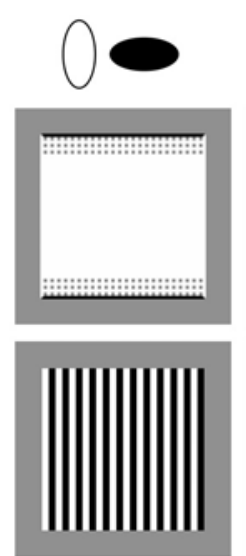

Image
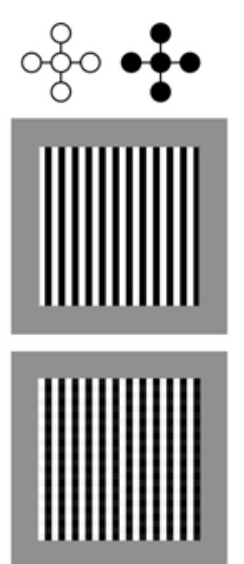

$\bigcirc$
(C)
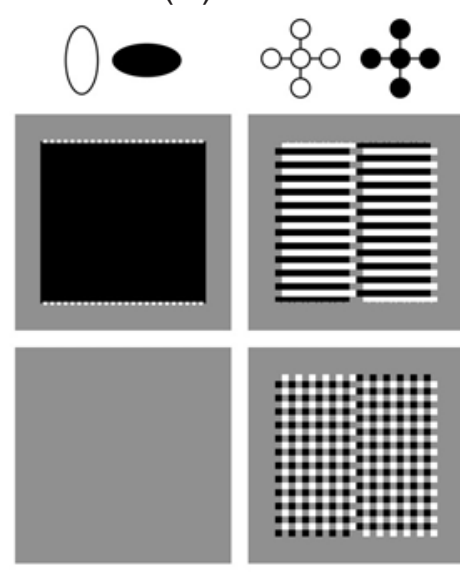

Image
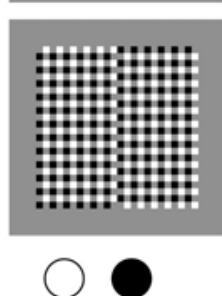

Figure 12. Simulation results for a sequence of images when the first image includes a split grating, using the new fillingin mechanism. (A) Model behavior during presentation of a split horizontal grating. (B) Model behavior during the last of the flickering vertical bar gratings. (C) Model behavior 1 sec after offset of the vertical bar grating. The model behavior matches the results of Experiment 2. 
ment validated a prediction generated by the FACADE model. As predicted, an inducing grid could produce a vertical or horizontal MCAI, depending on the orientation of the second inducing bar grating. This finding validates the explanation of MCAIs proposed by Francis and Rothmayer and also emphasizes the importance of filling-in for explaining these percepts.

The second experiment further tested the properties of filling-in the FACADE model. The model predicted that a split grating should not produce a visible MCAI, but the experimental data contradicted this prediction. We suggested that the fault in the model was due to its use of an extrapolating diffusive type of filling-in mechanism and proposed an alternative mechanism that could extrapolate when boundary signals are not present and interpolate when boundary signals are present. This switch in filling-in behavior seems to be necessary to account for the MCAIs produced by split gratings. This is a significant finding. Filling-in has been a key part of theories of visual perception for nearly 50 years, and a diffusive spreading of information has been the standard mechanism to support filling-in. The properties of MCAIs, while dependent on filling-in, suggest that diffusion is not a valid mechanism, at least within the FACADE model.

Despite the demonstrated need for a new filling-in mechanism, we reiterate the conclusion of Francis and Rothmayer (2003) that any system capable of accounting for the percepts of MCAIs needs to produce orientation afterresponses and color afterresponses, and combine them at a filling-in stage to form a surface representation. We believe that if the ideas proposed by Vidyasagar et al. (1999), for example, were elaborated to a degree that allowed for the creation of a simulation at the level we have provided here, the resulting model would look quite similar to the FACADE theory - namely, by the development of a stage for filling-in of surface percepts.

We believe that it is significant that the filling-in mechanism seems to have an interpolating property because this means its computational properties are similar to the BCS. Previous work on the FACADE model has suggested that boundary contour processing necessarily involves an interpolating process, and substantial development at both computational and neurophysiological levels has been proposed to support such a model (Grossberg et al., 2002; Grossberg \& Mingolla, 1985a, 1985b; Grossberg et al., 1997; Raizada \& Grossberg, 2003). We suggest that the same kind of processing will also be relevant for the processing of surface information. We expect that additional investigations of MCAIs will help guide the development of neural mechanisms for filling-in.

\section{REFERENCES}

ArRINGTON, K. F. (1994). The temporal dynamics of brightness fillingin. Vision Research, 34, 3371-3387.

Arrington, K. F. (1996). Directional filling-in. Neural Computation, 8, 300-318.

Brainard, D. H. (1997). The Psychophysics Toolbox. Spatial Vision, 10, 433-436.
Broerse, J., Vladusich, T., \& O'Shea, R. (1999). Colour at edges and colour spreading in McCollough effects. Vision Research, 39, 13051320.

Cohen, M. A., \& Grossberg, S. (1984). Neural dynamics of brightness perception: Features, boundaries, diffusion, and resonance. Perception \& Psychophysics, 36, 428-456.

DA Pos, O., \& BREssan, P. (2003). Chromatic induction in neon colour spreading. Vision Research, 43, 697-706.

Francis, G., Grossberg, S., \& Mingolla, E. (1994). Cortical dynamics of feature binding and reset: Control of visual persistence. Vision Research, 34, 1089-1104.

Francis, G., \& Rothmayer, M. (2003). Interactions of afterimages for orientation and color: Experimental data and model simulations. Perception \& Psychophysics, 65, 508-522.

Friedman, H. S., ZHOU, H., \& VON DER Heydt, R. (2003). The coding of uniform colour figures in monkey visual cortex. Journal of Physiology, 548, 593-613.

GERrITs, H., \& VENDRIK, A. (1970). Simultaneous contrast, filling-in process and information processing in man's visual system. Experimental Brain Research, 11, 431-447.

GrossberG, S. (1972). A neural theory of punishment and avoidance: II. Quantitative theory. Mathematical Biosciences, 15, 253-285.

Grossberg, S. (1987). Cortical dynamics of three-dimensional form, color, and brightness perception: I. Monocular theory. Perception \& Psychophysics, 41, 87-116.

GrossberG, S. (1994). 3-D vision and figure-reground separation by visual cortex. Perception \& Psychophysics, 55, 48-120.

Grossberg, S. (1997). Cortical dynamics of three-dimensional figurereground perception of two-dimensional figures. Psychological Review, 104, 618-658.

Grossberg, S., Hwang, S., \& Mingolla, E. (2002). Thalamocortical dynamics of the McCollough effect: Boundary-surface alignment through perceptual learning. Vision Research, 42, 1259-1286.

Grossberg, S., \& Kelly, F. J. (1999). Neural dynamics of binocular brightness perception. Vision Research, 39, 3796-3816.

Grossberg, S., \& McLoughlin, N. (1997). Cortical dynamics of 3-D surface perception: Binocular and half-occluded scenic images. Neural Networks, 10, 1583-1605.

Grossberg, S., \& Mingolla, E. (1985a). Neural dynamics of form perception: Boundary completion, illusory figures, and neon color spreading. Psychological Review, 92, 173-211.

Grossberg, S., \& Mingolla, E. (1985b). Neural dynamics of perceptual grouping: Textures, boundaries, and emergent segmentations. Perception \& Psychophysics, 38, 141-171.

Grossberg, S., Mingolla, E., \& Ross, W. D. (1997). Visual brain and visual perception: How does the cortex do perceptual grouping? Trends in Neurosciences, 20, 106-111.

Grossberg, S., \& Todorović, D. (1988). Neural dynamics of 1-D and 2-D brightness perception: A unified model of classical and recent phenomena. Perception \& Psychophysics, 43, 241-277.

Hong, S., \& Grossberg, S. (2003). Cortical dynamics of surface lightness anchoring, filling-in, and perception. Journal of Vision, 3, 415a, http://journalofvision.org/3/9/415/, doi:10.1167/3.9.415.

Hunter, W. (1915). Retinal factors in visual after-movement. Psychological Review, 22, 479-489.

Komatsu, H., Kinoshita, M., \& Murakami, I. (2000). Neural responses to the retinotopic representation of the blind spot in the Macaque V1 to stimuli for perceptual filling-in. Journal of Neuroscience, 20, 9310-9319.

Livingstone, M. S., \& Hubel, D. H. (1984). Anatomy and physiology of a color system in the primate visual cortex. Journal of Neuroscience, 4, 309-356.

MacKAY, D. (1957). Moving visual images produced by regular stationary patterns. Nature, 180, 849-850.

Neumann, H., Pessoa, L., \& Mingolla, E. (1998). A neural architecture of brightness perception: Non-linear contrast detection and geometry-driven diffusion. Image \& Vision Computing, 16, 423-446.

PARADISO, M. A., \& NAKAYAMA, K. (1991). Brightness perception and filling-in. Vision Research, 31, 1221-1236.

PELLI, D. G. (1997). The VideoToolbox software for visual psychophysics: Transforming numbers into movies. Spatial Vision, 10, 437-442. 
Pessoa, L., Mingolla, E., \& Neumann, H. (1995). A contrast- and luminance-driven multiscale network model of brightness perception. Vision Research, 35, 2201-2223.

Pessoa, L., Thompson, E., \& NoË, A. (1998). Finding out about fillingin: A guide to perceptual completion for visual science and the philosophy of perception. Behavioral \& Brain Sciences, 21, 723-756.

Pierce, A. H. (1900). The illusory dust drift: A curious optical phenomenon. Science, 12, 208-211.

Pinna, B., Brelstaff, G., \& Spillmann, L. (2001). Surface color from boundaries: A new watercolor illusion. Vision Research, 41, 2669-2676.

Purkinje, J. (1823). Beobachtungen und Versuche zur Physiologie der Sinne: Beiträge zur Kenntniss des Sehens in subjectiver Hinsichts. Prague: Calve.

RAIZADA, R., \& GRossberg, S. (2003). Towards a theory of the laminar architecture of cerebral cortex: Computational clues from the visual system. Cerebral Cortex, 13, 100-113.

RudD, M. E., \& Arrington, K. F. (2001). Darkness filling-in: A neural model of darkness induction. Vision Research, 41, 3649-3662.

Shimojo, S., Kamitani, Y., \& Nishida, S. (2001). Afterimage of perceptually filled-in surface. Science, 293, 1677-1680.

Stoper, A. E., \& Mansfield, J. G. (1978). Metacontrast and paracontrast suppression of a contourless area. Vision Research, 18, 1669 1674.

Todorović, D. (1987). The Craik-O'Brien-Cornsweet effect: New varieties and their theoretical implications. Perception \& Psychophysics, 42, 545-560.

Vidyasagar, T. R., Buzas, P., Kisyarday, Z. F., \& Eysel, U. T. (1999). Release from inhibition reveals the visual past. Nature, 399, 422-423. 


\section{APPENDIX \\ Simulations}

The simulations used to create Figures 2, 3, and 6 used the equations and procedures described in Francis and Rothmayer (2003). For the simulations reported in Figure 12, all but the equations for the filling-in process were the same as those in Francis and Rothmayer's study. The filling-in process for the new simulations is given below.

In our simulations, the FCS consists only of the filling-in stages. There are separate filling-in stages for white and black. We will describe the equations for the white filling-in stage. The equations are identical for the black filling-in stage, with only the terms for black and white switched. Each pixel in a filling-in stage has an activity value, which is designated as $W_{i, j}$ and $B_{i, j}$ for the white and black filling-in stages, respectively.

Because our stimuli are restricted to have only vertical and horizontal edges, the petals of the receptive field in Figure 11 were limited to only up, down, left, and right. Although we can hypothesize a variety of neural mechanisms to instantiate the equations we use, at the moment we are more interested in the algorithms and their effects. Because additional data constrains the model, we expect to be able to derive more specific mechanisms with neural components.

For each petal at position $(i, j)$, several terms were computed. Our discussion will describe the calculations for the up petal. The first term to be computed was the extent of sampling, $u_{i, j}$. This index was defined by starting at pixel position $(i, j)$ and moving up along a vertical path $(i, j-k)$ to find the first pixel location that had a nonzero horizontal boundary signal, $H_{i, j-k}$ and the last position to have a nonzero white activity, $W_{i, j-k}$. The extent of sampling by this petal was the closer of these two positions to the cell center. Written mathematically:

$$
u_{i, j}=\min \left[\min \left(k, \text { such that } H_{i, j-k}>0\right), \max \left(k, \text { such that } W_{i, j-k}>01\right)\right] .
$$

Sampling of activities by this subfield was then the weighted sum of differences between white and black activities from other cells in the filling-in stage.

$$
U_{i, j}=\sum_{k=1}^{u_{i, j}}\left(W_{i, j-k}-B_{i, j-k}\right)
$$

The definition of $u_{i, j}$ restricts $u_{i, j}$ from summing terms that are beyond a horizontal boundary or from summing terms that extend beyond the furthest white signal in the up receptive field subunit.

A similar calculation was made for every subfield. Finally, if two subfields had sampled from at least one pixel each, or if one subfield had sampled from at least one pixel and $W_{i, j}$ was greater than zero, a new value of $W_{i, j}$ was computed as the average sampled value across all subfields

$$
W_{i, j}=\frac{U_{i, j}+D_{i, j}+L_{i, j}+R_{i, j}+W_{i, j}}{u_{i, j}+d_{i, j}+l_{i, j}+r_{i, j}+1},
$$

where each uppercase letter indicates the sum of sampled inputs from each subfield and the lowercase letters indicate the number of sampled inputs from each subfield.

The net result is that each pixel in the filling-in stage computes the average difference between white and black cells among all sampled cells in the filling-in stages. The sampling is restricted by the presence of BCS boundary signals and by the physical arrangement of the signals themselves. If the BCS signals keep the black and white signals in separate FIDOs, the inhibition between filling-in stages will be zero, and the computed activity within a FIDO will be the average of values that feed into the filling-in stage.

The process involves feedback, which was implemented as an iterative computation. At initialization, we set $W_{i, j}=$ $w_{i, j}$ and $B_{i, j}=b_{i, j}$ for all $(i, j)$. We then computed equation (3) for every cell in the filling-in stages. These new values then replaced the old cell values, and equation (3) was recalculated. The process was stopped when the largest change in any cell's activity across an iteration was less than 0.1 .

Finally, each cell activity was thresholded so that any value less than 0.01 was set to zero. The values for black were subtracted from the values for white and normalized for plotting in the simulation figures.

(Manuscript received May 22, 2003;

revision accepted for publication June 10, 2004.) 